\title{
Review Article \\ Transcutaneous Electrical Stimulation and Dysphagia Rehabilitation: A Narrative Review
}

\author{
Ali Barikroo \\ Swallowing Physiology and Rehabilitation Research Laboratory, Speech Pathology and Audiology Program, Kent State University, \\ Kent, Ohio, USA \\ Correspondence should be addressed to Ali Barikroo; abarikro@kent.edu
}

Received 14 November 2019; Accepted 2 May 2020; Published 11 May 2020

Academic Editor: Richard Crevenna

Copyright ( 2020 Ali Barikroo. This is an open access article distributed under the Creative Commons Attribution License, which permits unrestricted use, distribution, and reproduction in any medium, provided the original work is properly cited.

Transcutaneous electrical stimulation (TES) was introduced as a modality for dysphagia rehabilitation more than a decade ago. The underlying premise of this modality is improving the structural movements and enhancing neural activation based on stimulationinduced muscle contractions. However, divisive evidence exists regarding the effectiveness of this treatment modality. This manuscript reviews current evidence regarding the effects of transcutaneous electrical stimulation (TES) on clinical and physiological aspects of swallowing function. Furthermore, this narrative review delineates the knowledge gap in this area and recommends future research roadmap. This review gives a comprehensive picture regarding current knowledge of TES to practicing speech and language pathologists and interested researchers. It highlights the need for more robust studies in this area. It also encourages researchers to focus more on the physiologic studies to understand the physiologic underpinning behind this treatment modality.

\section{Introduction}

Transcutaneous electrical stimulation (TES) has been used for several decades in other rehabilitation fields for a variety of therapeutic aims, such as strengthening muscles, increasing joint range of motion, preventing muscle atrophy, reducing pain, increasing sensory awareness, and enhancing the healing process [1]. TES is a reasonably new treatment modality in swallowing therapy, having been introduced in 2002 [2]. TES is purported to strengthen weak swallowing muscles and improve swallowing function in patients with dysphagia. Many studies have been conducted to understand the impact of TES on different aspects of swallowing function. In the following, the effects of TES on the clinical and physiological aspects of swallowing are being reviewed.

1.1. Effects of TES on Clinical Aspect of Swallowing. The effects of TES on the clinical aspects of swallowing rehabilitation have been studied to a significant extent but with conflicting outcomes. For example, an early study [2] reported superior clinical benefits of TES over conventional swallow- ing therapy in patients with dysphagia secondary to stroke. However, no randomization was performed for participants. Furthermore, no standard protocol was used to evaluate patients' function during videofluoroscopy. In a retrospective cohort study, Blumenfeld et al. [3] found superior treatment benefits for dysphagia therapy with TES compared to traditional dysphagia therapy in individuals in a long-term acute care facility. Nevertheless, the findings of this study should be considered in light of the inherent limitations of a retrospective cohort design, such as a lack of control over confounding factors and investigators' bias. In a case-control study, Kushner et al. [4] compared the effect of TES combined with traditional dysphagia therapy and traditional dysphagia therapy alone following stroke, reporting significantly enhanced outcomes for the combined therapy approach. Again, the findings of this study should be interpreted in the context of its limitations, such as a nonrandomized procedure for selecting the patients, the unequal size of the case and control groups, and no masking for the therapist and/or evaluator regarding the participant's assigned group. However, these same findings were later replicated in studies with 
stronger research designs $[5,6]$. Combining TES with other modalities has shown promising results in other neurologic populations as well. For example, Tang et al. [7] showed that combining TES with surface electromyography (sEMG) can improve swallowing function, nutritional status, and airway safety in patients with Alzheimer's disease. Finally, Chen et al. [8], in a meta-analysis of eight randomized and quasirandomized controlled trials, reported that using TES combined with other swallowing therapy approaches is potentially more effective than using TES alone for treating patients with poststroke dysphagia. However, they stated a lack of evidence supporting TES apart as superior to swallowing therapy. Apart from the peripheral impact on improving the strength of swallowing muscles (i.e., peripheral contribution pathway), TES may also have the potential to treat dysphagia through enhancing CNS sensory input [9]. This resonated sensory input can increase neural activation in CNS circuits, which can facilitate motor unit recruitment (i.e., central contribution pathway) [10]. In this regard, a recent study has suggested that combining TES with bilateral repetitive transcranial magnetic stimulation (rTMS) can lead to higher cortical activation and better swallowing function in patients with stroke [11].

Contrary to these positive clinical outcomes for TES, some studies have reported equivocal or weaker clinical benefits for TES-based dysphagia treatment compared with traditional therapy. For example, two similar randomized clinical trials in patients with dysphagia secondary to Parkinson's disease $[12,13]$ reported no enhanced clinical benefit of TES when combined with traditional dysphagia therapy. Using a meta-analysis, Tan et al. [14] reported that TES was comparable to conventional dysphagia therapy following stroke. Some studies have compared the effect of TES on swallowing with other exercise-based programs. GuillénSolà et al. [15] compared the impact of inspiratory/expiratory muscle strength training with TES, and traditional treatment on swallowing function. The findings of this study indicated that both groups $\mathrm{A}$ and $\mathrm{B}$ had comparable impacts on improving the swallowing safety. In a randomized clinical trial, Carnaby et al. [16] compared three treatment approaches in patients with dysphagia: traditional dysphagia therapy, McNeill Dysphagia Therapy Program (MDTP) with sham TES, and MDTP with TES. The results indicated that TES did not improve the effect of MDTP; instead, MDTP with TES showed fewer clinical benefits than MDTP with sham TES. In a double-blinded randomized clinical trial, Langmore et al. [17] compared two therapy approaches in patients with dysphagia following head and neck cancers: TES plus swallowing exercises versus sham TES plus swallowing exercises. This study demonstrated no added benefit of TES when combined with traditional swallowing exercises. Instead, based on the penetration aspiration scale, patients with sham TES experienced safer swallows than patients with TES.

In summary, a great deal of controversy exists in the literature regarding the clinical effectiveness of the existing TES protocols. While these studies vary significantly in quality and methodology, the majority of the studies reported that using the existing TES protocol alone might not have the desired clinical effects on the swallowing system. Alternatively, they described that combining the current TES protocol with other dysphagia rehabilitation techniques has induced better clinical outcomes. Table 1 summarizes the published studies regarding the effect of TES on the clinical aspect of swallowing.

1.2. Effects of TES on Physiological Aspects of Swallowing. A modest amount of evidence exists regarding the physiological impact of TES on swallowing muscles. One group of studies focused on the immediate effect of TES on the kinematic aspect of swallowing. For example, Ludlow et al. [18] applied TES on both submental and infrahyoid areas in 11 patients with dysphagia, indicating an immediate lowering effect of TES selectively on the hyoid bone at rest. However, this study was limited only to a small sample of patients and was underpowered. In a larger study, Humbert et al. [19] studied the effect of 10 electrode placements on a hyolaryngeal excursion at rest and during swallowing in 29 healthy adults. In an extension to Ludlow et al.'s study, they reported not only a descending pattern for the hyoid bone both at rest and during swallowing but also for the larynx. This descending effect of TES has been referred to the contraction of hyolaryngeal depressor muscles. These muscles are larger and closer to the neck surface compared with the hyolaryngeal elevator muscles. As a result of this anatomy, stimulation of the infrahyoid area primarily impact hyolaryngeal depressor muscles, thereby pulling down the hyolaryngeal complex. In a similar study, Kim et al. [20] tested the effects of three different electrode placements on hyolaryngeal positions in 20 healthy adults and found similar descending patterns for both the hyoid and larynx at rest for all three different electrode placements. In another study, Lee et al. [21] compared the immediate effect of two electrode placements (both submental and infrahyoid regions versus submental placement alone) on hyolaryngeal position in 15 patients with dysphagia. The findings of this study were in line with the majority of previous studies indicating an immediate lowering effect of TES on the hyolaryngeal complex during stimulation of both submental and infrahyoid regions. Given the immediate descending effect of TES on hyolaryngeal excursion, Park et al. [22] used effortful swallow as an exercise paradigm against the resistance of lowered hyoid under TES in healthy adults. The results of this study demonstrated that superior hyoid excursion increased after two weeks. Nam et al. [23] later replicated this study paradigm in dysphagia patients with brain injury and reported similar findings. Beyond this demonstrated impact of existing TES protocol on swallowing kinematics, few studies have investigated the immediate impact of TES on other swallowing physiology measures. For example, using conventional manometry, Heck et al. [24] reported no immediate effect of TES on pharyngeal pressure in 27 healthy young adults. Nevertheless, they described delayed effects of TES that manifested as decreased hypopharyngeal pressure and increased upper esophageal sphincter (UES) relaxation, which continued for up to one hour after stimulation. Given the lack of immediate pharyngeal pressure response (i.e., peripheral effect), the authors have assumed the observed delayed effect is centrally driven by 


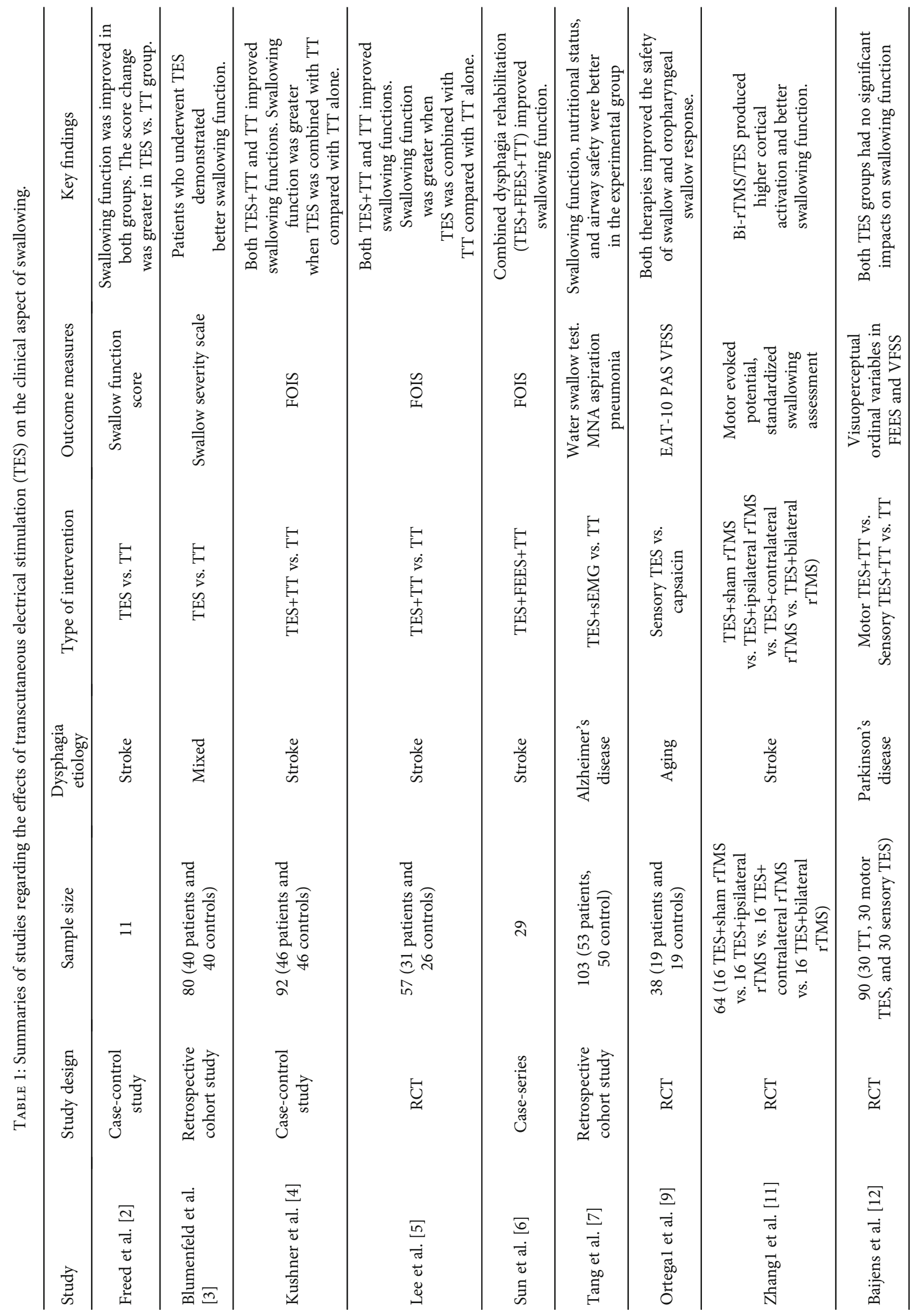




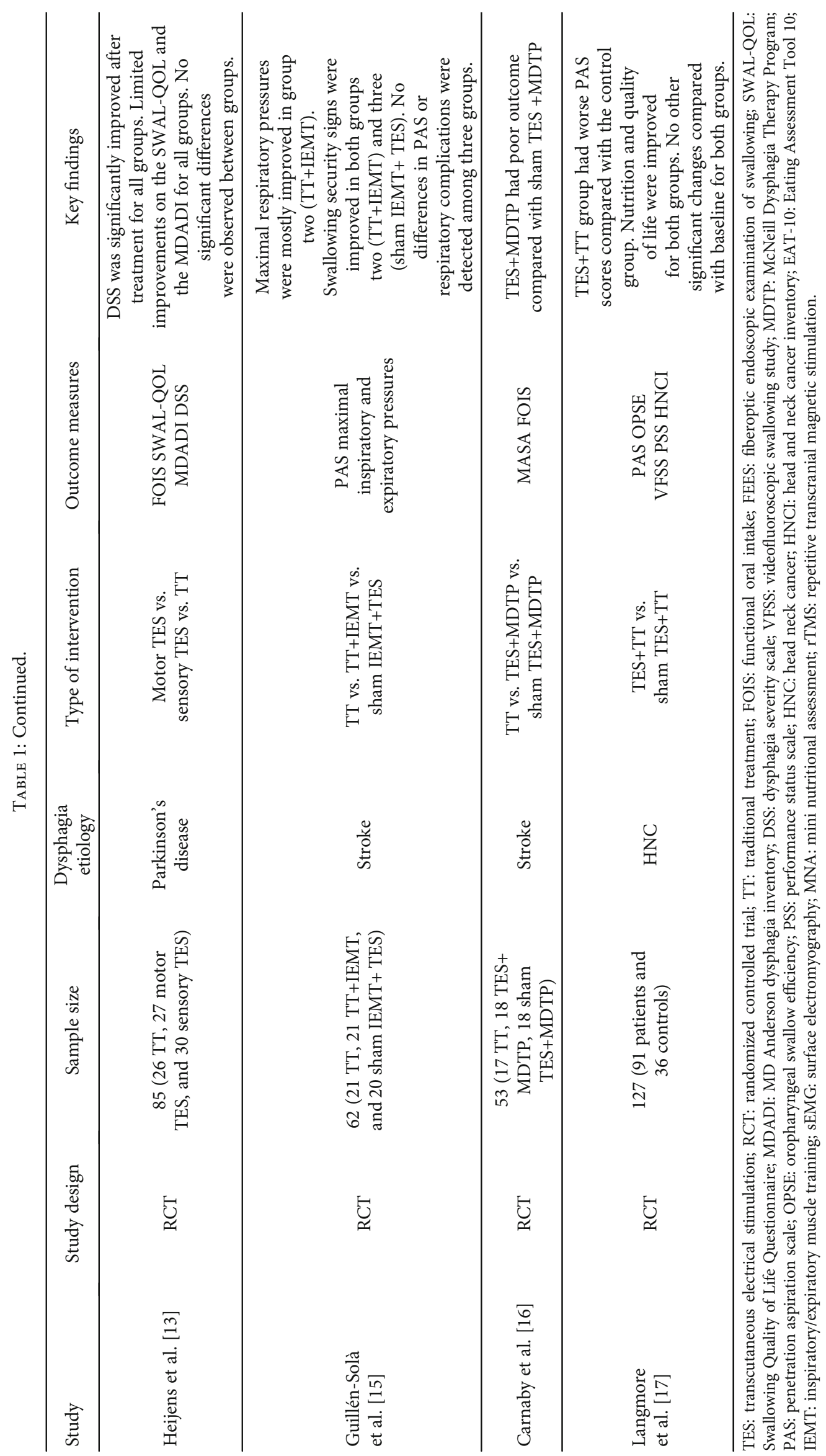


the brainstem (i.e., central effect). In a forward step, some studies investigated the effect of different TES parameters on swallowing function. In this regard, Berretin-Felix et al. [25] reported differential effects of sensory versus motor TES amplitudes on lingual-palatal and pharyngeal pressure in healthy young versus older adults. The results of this study demonstrated that high-amplitude TES reduced anterior and posterior lingua-palatal peak pressures but increased hypopharyngeal peak pressures for both age groups. Lowamplitude TES increased the base of tongue (BOT) peak pressures in older adults but reduced BOT peak pressures in younger adults. Similarly, in a secondary analysis of archived pressure data, Barikroo et al. [26] reported differential effects of TES amplitudes on lingual-palatal and pharyngeal pressure timing across age groups. Specifically, they reported no immediate effect of high-amplitude TES on lingual-palatal and pharyngeal pressure timing during swallowing for both healthy young and older adults. Conversely, they reported faster pharyngeal pressure during swallowing for older adults following low-amplitude TES. Recently, new studies have focused on optimizing the TES protocol for swallowing. For example, Barikroo et al. [27] showed that using short pulse duration $(300 \mu \mathrm{s})$ compare with long pulse duration $(700 \mu \mathrm{s})$ could further increase the maximum amplitude tolerance (MAT) without increasing the perceived discomfort level. The authors concluded that increasing MAT after using short pulse duration potentially stimulates deeper swallowing muscles. In a follow-up study, the same group of authors tested the effect of submental TES with varying pulse durations on lingual-palatal pressure measures during swallowing. They reported that using short pulse duration significantly decreased lingual-palatal pressure compared with long pulse duration [28]. This finding was also replicated in another study. Specifically, Takahashi et al. [29] investigated the effect of laryngeal TES with short pulse duration $(200 \mu \mathrm{s})$ on swallowing performance. They reported decreased tongue pressure and hyoid elevation during swallowing. These studies may suggest that using TES with short pulse duration may help us in triggering swallowing muscles that are buried in deeper tissue layers such as genioglossus, hyoglossus, and thyrohyoid. Pulse frequency is another TES parameter that impacts the quality of muscle contractions and likely swallowing physiology. The pulse frequency is associated with modulating the firing rate of motor unit recruitments and the strength of muscle contractions [30]. The majority of existing TES protocols use an $80 \mathrm{~Hz}$ frequency to stimulate these muscles. This frequency potentially fatigues the swallowing muscles, which should typically be stimulated at $30 \mathrm{~Hz}$ [31]. Furthermore, some studies suggest that using a pulse frequency at the range of $1-120 \mathrm{~Hz}$ induces greater discomfort when compared with kilohertz frequencies. Moreover, kilohertz frequencies induce less skin impedance and can penetrate deeper through the tissues [32]. In this regard, Jungheim et al. [33] utilized high-resolution manometry to compare the effect of two TES protocols with $2700 \mathrm{~Hz}$ and $100 \mathrm{~Hz}$ on swallowing pressure. The findings of this study indicated that the TES protocol with kilohertz frequency increased the base of tongue retraction. However, no significant effect of frequency was observed on the upper esophageal sphincter opening. Additional studies are needed to understand the effect of varying TES frequency on fatigue and swallowing function. One of the other TES parameters that need to be explored is the electrical stimulation waveform. Current TES waveforms for swallow rehabilitation apply square waves [34-38]. However, literature relating to other parts of the body indicates that a sine waveform has deeper penetration through human tissue [37-45], which can be beneficial if we need to target deep swallowing muscles. It is now clear that a one-size-fits-all approach for TES-based dysphagia rehabilitation is simplistic. Therefore, the TES parameters should be tailored based on the pathophysiology of swallowing. More studies are needed to understand the effect of other TES parameters on swallowing physiology.

In summary, the majority of studies recommend that applying TES on submental and infrahyoid regions lowers the tongue and hyolaryngeal complex. Furthermore, some studies have indicated that this TES-induced hyolaryngeal descending effect can be used as a resistive exercise paradigm to improve the tongue strength and hyolaryngeal excursion during swallowing. Beyond the kinematic effect, recent evidence suggests that various TES amplitude levels may have a distinctive modulation impact on the swallowing physiology across age groups. Specifically, older adults benefit more from certain TES amplitudes than younger adults in some measured physiologic activities and more significant deterioration in other aspects of swallowing physiology. Furthermore, using short pulse duration may increase maximum amplitude tolerance that can subsequently increase the depth of electrical current penetration. Moreover, the lack of information exists regarding the effect of pulse frequency on swallowing physiology. Table 2 summarizes the published literature regarding the effect of TES on the physiological aspect of swallowing.

\section{Conclusions and Future Research Directions}

The gist of conducted clinical trials suggests that TES works best as an adjunct modality when it is combined with other rehabilitation techniques. In addition, it seems that TESbased dysphagia rehabilitation has been more successful in a certain group of etiologies (i.e., stroke) than the others (i.e., head and neck cancer, progressive neurological disorders). However, this message has not been consistent across different studies. Part of this controversy may relate to the weak methodological designs for many of the performed studies such as small sample size, inadequate patient selection criteria, lack of control group, lack of control over confounding variables, short-term follow-up periods, and use of nonstandardized tests for evaluating outcome measures. Thus, having robust multicenter clinical trials can help us to have a clear picture regarding the effectiveness of this modality alone or in combination with other treatment modalities across different etiologies. Furthermore, it is essential to incorporate the results of physiological studies into clinical trials. That is what I call a physiological-based TES rehabilitation approach. Based on this approach, the underlying physiological issues of swallowing should be 


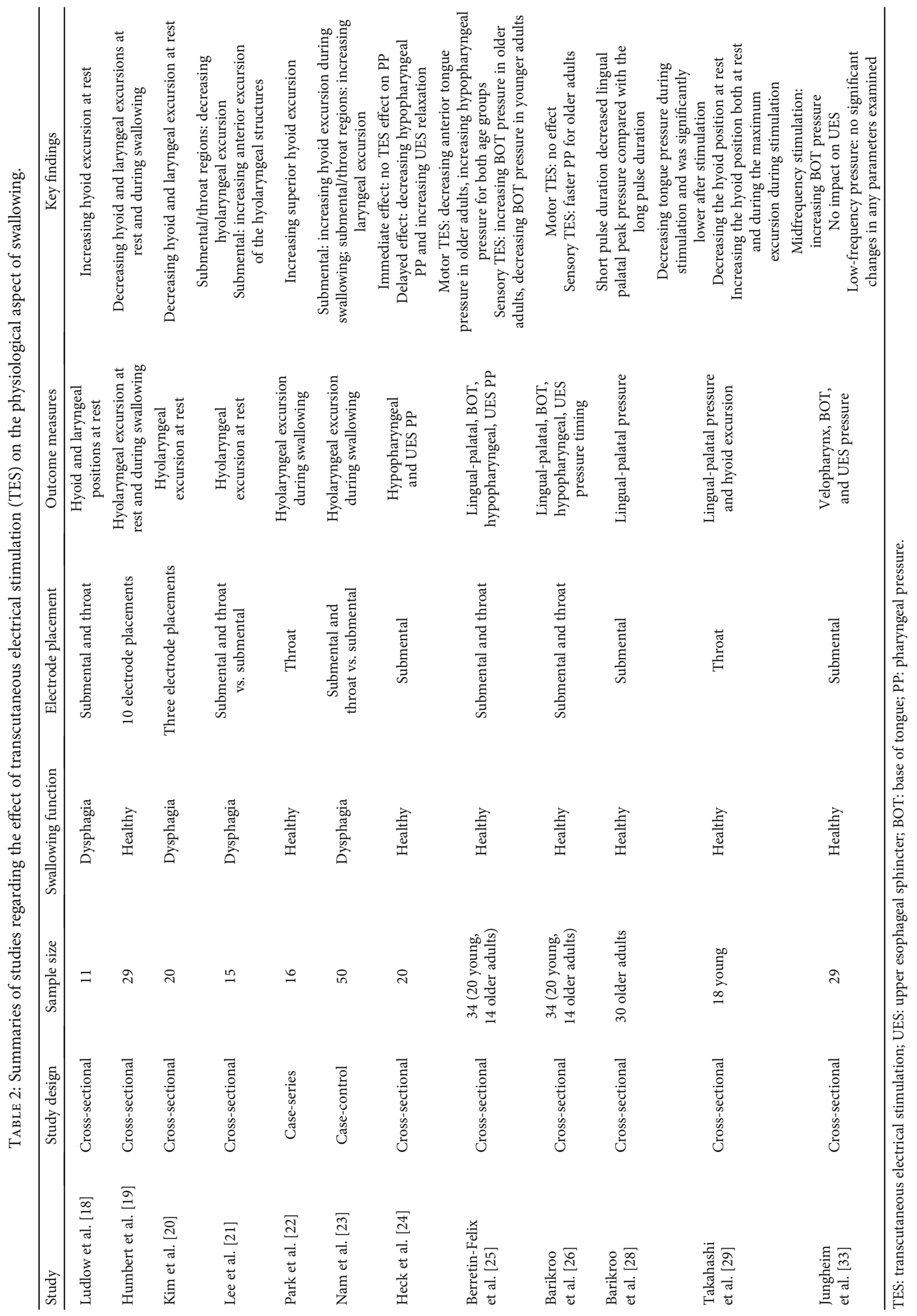


identified first (i.e., lower hyolaryngeal excursion, decreased base of tongue pressure, or delayed laryngeal vestibule closure), and the TES protocol should be adjusted in a way to address that specific pathophysiology. As a result, more studies are required to understand the effect of TES on different aspects of swallowing physiology. Many clinical studies were conducted with preset TES parameters (i.e., electrode size and placement, waveform, amplitude, frequency, and pulse duration) with no information regarding the rationale behind their decision. It is now clear that a one-size-fits-all approach for TES-based dysphagia rehabilitation is misdirected. Each TES parameter has the potential to change the quality of swallowing muscle contractions, which may impact on the physiology of swallowing distinctively and ultimately alter the treatment outcomes. Beyond the effect of TES parameters, biopsychological characteristics of each patient, such as subcutaneous adipose tissue thickness, pain sensitivity, and fear of pain may modify the effects of TES on swallowing function. As a result, more studies are needed to understand the combined effect of different TES parameters and biopsychological factors on swallowing physiology and dysphagia rehabilitation. Finally, additional studies are required to understand the impact of TES on neural activation and neural plasticity in patients with varying etiology.

\section{Conflicts of Interest}

The author declares that there is no conflict of interest regarding the publication of this paper.

\section{Acknowledgments}

The dissertation of the manuscript was presented to the graduate school of the University of Florida in partial fulfillment of the requirements for the degree of doctor of philosophy titled as "Comparing The Effects Of A Novel Versus Existing Transcutaneous Electrical Stimulation Protocol On Lingual Palatal Peak Pressure And Pressure Duration During Swallowing In Healthy Older Adults".

\section{References}

[1] B. M. Doucet, A. Lam, and L. Griffin, "Neuromuscular electrical stimulation for skeletal muscle function,", The Yale Journal of Biology and Medicine, vol. 85, no. 2, pp. 201-215, 2012.

[2] M. L. Freed, L. Freed, R. L. Chatburn, and M. Christian, "Electrical stimulation for swallowing disorders caused by stroke," Respiratory Care, vol. 46, no. 5, pp. 466-474, 2001.

[3] L. Blumenfeld, Y. Hahn, A. LePage, R. Leonard, and P. C. Belafsky, "Transcutaneous electrical stimulation versus traditional dysphagia therapy: a nonconcurrent cohort study," Otolaryngology-Head and Neck Surgery, vol. 135, no. 5, pp. 754757, 2016.

[4] D. S. Kushner, K. Peters, S. T. Eroglu, M. Perless-Carroll, and D. Johnson-Greene, "Neuromuscular electrical stimulation efficacy in acute stroke feeding tube-dependent dysphagia during inpatient rehabilitation," American Journal of Physical Medicine \& Rehabilitation, vol. 92, no. 6, pp. 486-495, 2013.

[5] K. W. Lee, S. B. Kim, J. H. Lee, S. J. Lee, J. W. Ri, and J. G. Park, "The effect of early neuromuscular electrical stimulation ther- apy in acute/subacute ischemic stroke patients with dysphagia," Annals of Rehabilitation Medicine, vol. 38, no. 2, pp. 153-159, 2014.

[6] S. F. Sun, C. W. Hsu, H. S. Lin et al., "Combined neuromuscular electrical stimulation (NMES) with fiberoptic endoscopic evaluation of swallowing (FEES) and traditional swallowing rehabilitation in the treatment of stroke-related dysphagia," Dysphagia, vol. 28, no. 4, pp. 557-566, 2013.

[7] Y. Tang, X. Lin, X. J. Lin et al., "Therapeutic efficacy of neuromuscular electrical stimulation and electromyographic biofeedback on Alzheimer's disease patients with dysphagia," Medicine (Baltimore), vol. 96, no. 36, article e8008, 2017.

[8] Y.-W. Chen, K.-H. Chang, H.-C. Chen, W.-M. Liang, Y.H. Wang, and Y.-N. Lin, "The effects of surface neuromuscular electrical stimulation on post-stroke dysphagia: a systemic review and meta-analysis," Clinical Rehabilitation, vol. 30, no. 1, pp. 24-35, 2015.

[9] O. Ortega, L. Rofes, A. Martin, V. Arreola, I. López, and P. Clavé, "A comparative study between two sensory stimulation strategies after two weeks treatment on older patients with oropharyngeal dysphagia," Dysphagia, vol. 31, no. 5, pp. 706716, 2016.

[10] A. J. Bergquist, J. M. Clair, O. Lagerquist, C. S. Mang, Y. Okuma, and D. F. Collins, "Neuromuscular electrical stimulation: implications of the electrically evoked sensory volley," European Journal of Applied Physiology, vol. 111, no. 10, pp. 2409-2426, 2011.

[11] C. Zhang, X. Zheng, R. Lu, W. Yun, H. Yun, and X. Zhou, "Repetitive transcranial magnetic stimulation in combination with neuromuscular electrical stimulation for treatment of post-stroke dysphagia," The Journal of International Medical Research, vol. 47, no. 2, pp. 662-672, 2019.

[12] L. W. J. Baijens, R. Speyer, V. L. Passos et al., "Surface electrical stimulation in dysphagic Parkinson patients: a randomized clinical trial," Laryngoscope, vol. 123, no. 11, pp. E38-E44, 2013.

[13] B. J. Heijnen, R. Speyer, L. W. J. Baijens, and H. C. A. Bogaardt, "Neuromuscular electrical stimulation versus traditional therapy in patients with Parkinson's disease and oropharyngeal dysphagia: effects on quality of life," Dysphagia, vol. 27, no. 3, pp. 336-345, 2012.

[14] C. Tan, Y. Liu, W. Li, J. Liu, and L. Chen, "Transcutaneous neuromuscular electrical stimulation can improve swallowing function in patients with dysphagia caused by non-stroke diseases: a meta-analysis," Journal of Oral Rehabilitation, vol. 40, no. 6, pp. 472-480, 2013.

[15] A. Guillén-Solà, M. M. Sartor, N. B. Soler, E. Duarte, M. C. Barrera, and E. Marco, "Respiratory muscle strength training and neuromuscular electrical stimulation in subacute dysphagic stroke patients: a randomized controlled trial," Clinical Rehabilitation, vol. 31, no. 6, pp. 761-771, 2016.

[16] G. Carnaby, L. LaGorio, M. Crary, and D. Miller, "A randomized double blind trial of neuromuscular electrical stimulation + McNeill dysphagia therapy (MDTP) after stroke (ANSRS). Presentation to the Dysphagia Research Society Annual Meeting. Toronto, Canada," Dysphagia, vol. 27, no. 4, pp. 569-620, 2012.

[17] S. E. Langmore, T. M. McCulloch, G. P. Krisciunas et al., "Efficacy of Electrical Stimulation and Exercise for Dysphagia in Patients with Head and Neck Cancer: A Randomized Clinical Trial," Head \& Neck, vol. 38, Supplement 1, pp. E1221-E1231, 2016. 
[18] C. L. Ludlow, I. Humbert, K. Saxon, C. Poletto, B. Sonies, and L. Crujido, "Effects of surface electrical stimulation both at rest and during swallowing in chronic pharyngeal dysphagia," Dysphagia, vol. 22, no. 1, pp. 1-10, 2007.

[19] I. A. Humbert, C. J. Poletto, K. G. Saxon et al., "The effect of surface electrical stimulation on hyolaryngeal movement in normal individuals at rest and during swallowing," Journal of Applied Physiology (Bethesda, MD: 1985), vol. 101, no. 6, pp. 1657-1663, 2006.

[20] S. H. Kim, B. M. Oh, T. R. Han, H. J. Jeong, and Y. J. Sim, "Different movement of Hyolaryngeal structures by various application of electrical stimulation in Normal individuals," Annals of Rehabilitation Medicine, vol. 39, no. 4, pp. 535-544, 2015.

[21] H. Y. Lee, J. S. Hong, K. C. Lee, Y. K. Shin, and S. R. Cho, "Changes in hyolaryngeal movement and swallowing function after neuromuscular electrical stimulation in patients with dysphagia," Annals of Rehabilitation Medicine, vol. 39, no. 2, pp. 199-209, 2015.

[22] J. W. Park, J. C. Oh, H. J. Lee, S. J. Park, T. S. Yoon, and B. S. Kwon, "Effortful swallowing training coupled with electrical stimulation leads to an increase in hyoid elevation during swallowing," Dysphagia, vol. 24, no. 3, pp. 296-301, 2009.

[23] H. S. Nam, J. Beom, B. M. Oh, and T. R. Han, "Kinematic effects of hyolaryngeal electrical stimulation therapy on hyoid excursion and laryngeal elevation," Dysphagia, vol. 28 , no. 4, pp. 548-556, 2013.

[24] F. M. Heck, S. H. Doeltgen, and M. L. Huckabee, "Effects of submental neuromuscular electrical stimulation on pharyngeal pressure generation," Archives of Physical Medicine and Rehabilitation, vol. 93, no. 11, pp. 2000-2007, 2012.

[25] G. Berretin-Felix, I. Sia, A. Barikroo, G. D. Carnaby, and M. A. Crary, "Immediate effects of transcutaneous electrical stimulation on physiological swallowing effort in older versus young adults," Gerodontology, vol. 33, no. 3, pp. 348-355, 2016.

[26] A. Barikroo, G. Berretin-Felix, G. Carnaby, and M. Crary, "Effect of transcutaneous electrical stimulation amplitude on timing of swallow pressure peaks between healthy young and older adults," Gerodontology, vol. 34, no. 1, pp. 24-32, 2017.

[27] A. Barikroo, G. Carnaby, D. Bolser, R. Rozensky, and M. Crary, "Transcutaneous electrical stimulation on the anterior neck region: the impact of pulse duration and frequency on maximum amplitude tolerance and perceived discomfort," Journal of Oral Rehabilitation, vol. 45, no. 6, pp. 436-441, 2018.

[28] A. Barikroo, K. Hegland, G. Carnaby, D. Bolser, T. Manini, and M. Crary, "The Effects of Electrical Stimulation Pulse Duration on Lingual Palatal Pressure Measures during Swallowing in Healthy Older Adults," Dysphagia, vol. 34, no. 4, pp. 529-539, 2019.

[29] K. Takahashi, K. Hori, H. Hayashi et al., "Immediate effect of laryngeal surface electrical stimulation on swallowing performance," Journal of Applied Physiology (Bethesda, MD: 1985), vol. 124, no. 1, pp. 10-15, 2018.

[30] C. S. Bickel, C. M. Gregory, and J. C. Dean, "Motor unit recruitment during neuromuscular electrical stimulation: a critical appraisal," European Journal of Applied Physiology, vol. 111, no. 10, pp. 2399-2407, 2011.

[31] C. L. Ludlow, "Electrical Stimulation Treatment," in Principles of Deglutition: A Multidisciplinary Text for Swallowing and its Disorders, R. Shaker, Ed., pp. 809-820, (C) Springer Science +Business Media, 2013.
[32] A. R. Ward, "Electrical stimulation using kilohertz-frequency alternating current," Physical Therapy, vol. 89, no. 2, pp. 181-190, 2009.

[33] M. Jungheim, C. Schubert, S. Miller, and M. Ptok, "Swallowing function after continuous neuromuscular electrical stimulation of the submandibular region evaluated by highresolution manometry," Dysphagia, vol. 32, no. 4, pp. 501508, 2017.

[34] H. Clark, C. Lazarus, J. Arvedson, T. Schooling, and T. Frymark, "Evidence-based systematic review: effects of neuromuscular electrical stimulation on swallowing and neural activation," American Journal of Speech-Language Pathology, vol. 18, no. 4, pp. 361-375, 2009.

[35] G. D. Carnaby-Mann and M. A. Crary, "Examining the evidence on neuromuscular electrical stimulation for swallowing," Archives of Otolaryngology - Head \& Neck Surgery, vol. 133, no. 6, pp. 564-571, 2007.

[36] G. Carnaby and A. Madhavan, "A systematic review of randomized controlled trials in the field of dysphagia rehabilitation," Current Physical Medicine and Rehabilitation Reports, vol. 1, no. 4, pp. 197-215, 2013.

[37] J. Petrofsky, "The effect of the subcutaneous fat on the transfer of current through skin and into muscle," Medical Engineering \& Physics, vol. 30, no. 9, pp. 1168-1176, 2008.

[38] J. Petrofsky, M. Laymon, M. Prowse, S. Gunda, and J. Batt, "The transfer of current through skin and muscle during electrical stimulation with sine, square, Russian and interferential waveforms," Journal of Medical Engineering \& Technology, vol. 33, no. 2, pp. 170-181, 2009.

[39] J. M. Clair-Auger, D. F. Collins, and J. P. A. Dewald, "The effects of wide pulse neuromuscular electrical stimulation on elbow flexion torque in individuals with chronic hemiparetic stroke," Clinical Neurophysiology, vol. 123, no. 11, pp. 22472255, 2012.

[40] A. S. Gorgey, E. Mahoney, T. Kendall, and G. A. Dudley, "Effects of neuromuscular electrical stimulation parameters on specific tension," European Journal of Applied Physiology, vol. 97, no. 6, pp. 737-744, 2006.

[41] O. Lagerquist and D. F. Collins, "Influence of stimulus pulse width on M-waves, H-reflexes, and torque during tetanic low-intensity neuromuscular stimulation," Muscle \& Nerve, vol. 42, no. 6, pp. 886-893, 2010.

[42] Y. Laufer and M. Elboim, "Effect of burst frequency and duration of kilohertz-frequency alternating currents and of lowfrequency pulsed currents on strength of contraction, muscle fatigue, and perceived discomfort," Physical Therapy, vol. 88, no. 10, pp. 1167-1176, 2008.

[43] L. Mesin, E. Merlo, R. Merletti, and C. Orizio, "Investigation of motor unit recruitment during stimulated contractions of tibialis anterior muscle," Journal of Electromyography and Kinesiology, vol. 20, no. 4, pp. 580-589, 2010.

[44] J. P. Reilly and H. Antoni, Electrical Stimulation and Electropathology, Cambridge University Press, 1992.

[45] W. B. Scott, J. B. Causey, and T. L. Marshall, "Comparison of maximum tolerated muscle torques produced by 2 pulse durations," Physical Therapy, vol. 89, no. 8, pp. 851-857, 2009. 